\title{
LAS DIATOMITAS MEXICANAS Y SU EMPLEO INDUSTRIAL
}

(Trabajo presentado por el autor en la Sesión de la Convención Geológica Nacional Correspondiente del 10. al 4 de junio de 195:)

J. Ariel Hernández Velasco $0^{*}$

\section{R E S U M E N}

En los últimos treinta años la atención dedicada al conocimiento y explotación de los minerales no metálicos ha sido muy eventual, por lo que no se conocen en forma definida los recursos con que cuenta el país en este aspecto de la minería. La diatomita, mineral no metálico, ya era conocida desde los tiempos más antiguos y algunas de las localidades existentes en el centro de la Repáblica han sido explotadas hasta nuestros días, explotación que en general ha seguido métodos rudimentarios, lo que ha traido como consecuencia el poco desarrollo de la industria.

Con objeto de ver las posibilidades que pudieran tener las diatomitas mexicanas y el de conocer las especies de diatomeas que las constituyen, se estudiaron cuatro muestras procedentes del Estado de México. Para el estudio de clasificación se tomaron como guía trabajos ejecutados por investigadores mexicanos y extranjeros.

En las muestras estudiadas se reconocieron las especies de diatomeas elasificadas con el nombre de: Achnanteas, Cymbeleas, Naviculeas, Surileleas, Sinedreas, Fragilareas y Gailoneleas.

La pureza química, limpieza de material, forma, tamaño, poder de absoreión y su poder filtrante (?), sugieren grandes posibilidades para ser empleada como material filtrante o como portador.

Muy bien podrían iniciarse trabajos de explotación e industrialización de algunos yacimientos de diatomitas que se localizan en las cercanías de la Ciudad de México; con este objeto se hace necesario un estudio más profundo de las posibilidades geológicoeconómicas y de el sistema más adecuado para explotar los yacimientos existentes en el Estado de México.

Una instalación en pequeño sería la base para iniciar la industrialización de las tierras diatomáceas, debiendo estar cons-

* Manuscrito recibido el 12 de agosto de 1954.

Químico y Mineralogista del Instituto para la Investigación de Recursos Minarales 
tituida por un horno secador, un molino primario desintegrador, cribas para separar impurezas, un horno de calcinación, un molino secundario, dos separadores de ciclón para partículas comprendidas entra 150 y 250 mallas y entre 250 y 325 mallas; adomás, se requieren los elementos necesarios para el trabajo que desempeña un laboratorio de análisis, molino de laboratorio, tamices standard, equipo adecuado para determinar el poder de filtración, poder de absorción, densidad aparente, grado de porosidad, grado de acidés $\mathrm{y}$, como complemento muy importante, un microscopio.

Tomando en cuenta el gran número de aplicaciones que tienen las tierras diatomáceas, el alto precio de importación de sus productos y el mercado existente, se recomienda a los señores industriales que diłijan su atención al estudio, explotación e industriá lización de las tierras diatomáceas, nuevo campo de actividades con brillantes perspectivas para el futuro.

\section{INTRODUCCION}

Aunque ya eran conocidos entre los indígenas el oro y el cobre, se ha señalado el año de 1521 como la fecha que malas ol nacimiento de la minería en México, época en que fueron descubiertas las minas de Taxco en el Estado de Guerrero, y el año de 1522 , la fecha en que se hicieron los primeros embarques de plata a Esspaña; desde entonces su economía, desarrollo o historia, han estado intimamente ligados al desenvolvimiento economico del país.

Un capítulo sumamente importante de la minería moderna $y$ que ha alcanzado notable desarrollo en el transeurso de los últimos treinta años, es el que se refiere al estudio y explotación de los minerales no metálicos, destacando los minerales extraídos $y$ beneficiados no por su contenido metálico, sino por sus propiedades físicas. No es necesario hacer hincapié, pues es de iodos conocido, que el desarrollo de la minería en nuestro país cólo comprende la explotación de los minerales nobles y sus asociados: plata, oro, plomo, zinc, y cobre, como explotaciones típicas desde la época colonial; $y$ en los últimos años, antimonio, fierro, arsónic, grafito, mercurio, tungsteno, fluorita y azufre, como minerales de explotación constante.

Realmente la industria mexicana se eneuentra en franco estado de desarrollo, por lo que los técnicos industriales ven un iuturo optimista; pero al dirigir nuestra atención hacia las actividades mineras $y$ en especial al conocimiento y explotación de los minerales no metálicos, vemos que la atención que se les ha concedido es tan pequeña, por no decir nula, que aún se desconoce ia existencia de los minerales no metálicos, y de los pocos yacimientos conocidos se carece de datos aprovechables por el industrial que 
pretende iniciar una nueva actividad; por ello desde el comienzo de sus trabajos encuentra problemas de muy difícil solución, fos que se transforman en barreras infranqueables que minan su voiuntad y economía.

En general, no digamos la explotación, sino el sonocimiento de Ios minerales no metálicos es nulo en el país y su explotación $y$ beneficio aún no forman parte de los trabajos de minería; pero es de suponer que las reservas de minerales no metálicos existentes son cuantiosas y de suma importancia para su desenvolvimiento económico,

En el año de 1917, el maestro Enrique Díaz Lozano, on bu trabajo sobre las diatomitas mexicanas (1), hace notar que la explotación de las tierras diatomáceas constituye una industria de brillantes perspectivas y a pesar de que en el país existen algunos yacimientos de importancia, a la fecha no es sino una industria rudimentaria. Treinta y cinco años más tarde, aún puede decirse que la explotación de las tierras diatomáceas es una in dustria de brillantes perspectivas y que, a excepción de la diatomita del Municipio de Tlaxcala, Estado de Tlaxcala, las posibilidades que puedan tener las demás localidades conocidas hasta ahora son desconocidas. Con este trabajo se pretende proporcionar mayor conocimiento de las tierras diatomáceas, dar a conocert su importancia y empleo, alentando al Industrial a buscas en nuestras propias reservas la materia prima mineral que importa.

Antes de iniciar la exposición del trabajo, el autor desea agradecer sinceramente la ayuda proporcionada por los señores David Varela, Félix Vértiz y Juan Díaz, por haber proporcionado las muestras necesarias y los datos relacionados con las mismas; af Instituto Nacional para la Investigación de Recursos Minerales se le agradece la oportunidad concedida para el desarrollo del trabajo y por último se agradece al Ing. Químico, señor Adaiberto Obregón, la colaboración y ayuda proporcionada en el desarrollo de los trabajos de análisis y control químico efectuados en cada una de las muestras.

\section{LAS DIATOMITAS O TIERRA DIATOMACEA}

La diatomita o tierra diatomacea ya era conocida desde tiempos remotos, aunque no por su constitución interna, sino más bien por su propiedades. Se habla de la preparación de losetas sumamente ligeras a partir de una tierra blanca $y$ se conocen algunos estucos que han resistido perfectamente la inclemencia del tiempo, sin que se observe aparentemente deterioro alguno (2). Nuestros indígenas conocían también este tipo de tierras y sus propiedades, las que aprovechaban en diversas formas (3). Natural-

(1) Bibliografía al firal de este trabajo. 
mente, hasta después del descubrimiento del microscopio se supo que estas tierras están constituidas por la aglomeración de remanentes fósiles de plantas acuáticas microsópicas, conocidas con el nombre de diatomeas.

Actualmente se le conoce por diversos nombres, lo que en ocasiones ha sido motivo de confusión; los nombres más comunes con que se le designa son: Tripolita, Tízar, Tizate (por los indígenas), Sil o Sel, Diealite, Filter Cel, Standard Super Cel, Hyflow Super Cel, Correspondiendo los últimos a nombres comerciales con give se le ha registrado. Erróneamente se le llama Trípoli, ya que éste es una forma de sílice, producto de alteración del pedernal 0 de calizas muy silícicas, que no contienen diatomeas. También crróneamente se le designa con el nombre de tierra de infusorios, ya que los infusorios pertenecen a un grupo con características diferentes a las de las diatomeas.

MINERALOGIA $(4,5,6)$--La diatomita es una variedad de ópalo, coloidal, amorfa, constituída por la acumulación de reston silícicos de diatomeas. Se presenta en forma de agregados muy finos y porosos, variando en su textura desde pobremente coherente hasta compacto. Sin crucero. Fractura paralela a los planos de sedimentación, concoidal o irregular. Quebradiza, frágil, se desm menuza fácilmente. Opaca. Dureza en terrones $H .=1 .-1.5$; en las partículas microscópicas $\mathrm{H}$. $=4.5-6.5$. Peso específico, Pe. verdadero $=1.9-2.35 ;$ Pe aparente, en bloques puros $\mathrm{y}$ se$\cos , \ldots 0.45 ;$ Pe en material puro e impalpable $=0.13$. Lustre de opaco a terroso. Color blanco, gris crema, canela, café y casi negro, ocasionalmente verdoso. Rayadura gris claro. Indice de rafacción variable $=1.42-1.48$. Insoluble en ácidos, a excepción del ácido fluorhídrico; soluble en álcalis (7). Absorbe agua desde 1.5 hasta 4 veces su peso. La conductividad térmica, cuando se encuentra en bloques secos con densidad aparente de 0.53 , varía desde $0.000227 \mathrm{Ca}$. Gr. Sec. a $200^{\circ} \mathrm{C}$, hasta 0.000315 Cal. Gr. Sec. a $800^{\circ} \mathrm{C}$. Para material pulverizado hasta el grado impalpable, con densidad aparente de 0.12 , varía desde 0.000127 Cal. Gi. Sec. a $200^{\circ} \mathrm{C}$ hasta 0.000379 Cal. Gr. Sec. a $800^{\circ} \mathrm{C}$.

Su composición química corresponde a la del ópalo, sílice $y$ agua de combinación, la que varía de 2 a $10 \%$. Se presenta acom. pañada de materia orgánica en cantidades variables desdo huellas hasta un $30 \%$, y cuntidades variables de impurezas como arena, sílice criptocristalina, arcilla, ceniza volcánica, carbonato de calcio, magnesio y sales solubles.

Los análisis de tierras diatomáceas, en sus determinaciones fundamentales, dan los siguientes resultados: 
Sílice $\left(\mathrm{SiO}_{2}\right)$

Alúmina $\left(\mathrm{Al}_{2} \mathrm{O}_{3}\right)$

Fierro total $\left(\mathrm{Fe}_{2} \mathrm{O}_{3}\right)$

Pérdidas por calcinación, agua, materia orgánica, anhídrido carbónico

\section{$72-92.7 \%$}

$2.5-9.5 \%$

Huellas - $3.3 \%$

LOCALIZACION. - Las tierras diatomáceas se encuentran como depósitos de extensiones variables constituyendo el fondo de lagunus, lagos, pantanos modernos o extinguidos, asociados con materia orgánica y materiales diversos de origen sedimentario, con espesores y dimensiones muy variables, concediéndoseles importancia industrial sólo a aquellos susceptibles de ser explotados económicamente.

Los depósitos pueden ser de origen marino o lacustre, de "yuas dulces o saladas y se consideran formados por la sedimentación o acumulación, bajo condiciones favorables de restos vegetales conocidos con el nombre de diatomeas. En relación con la edad geológica, varían dosde el Cretácico hasta ol Reciente. dosarrollándose con gran profusión particularmente durante el Mioceno, al que pertenecen los depósitos más importantes conotidos actualmente $y$ es probable que existan algunos en formación.

\section{CLASIFICACION Y DESCRIPCION DE LAS DIATOMEAS}

Las diătomeas han sido ampliamente estudiadas por ei Dr. J. Pelletan (8), quien presentó un trabajo de clasificación y descripción de las diatomeas. En los años subsecuentes distintos investigadores suecos y alemanes. En nuestro país, el maestro Enrique Díaz Lozano inició el estudio de las diatomeas existentes on el lago de Texcoco y las del Valle de Toxi, Estado de México $(9,10)$.

La familia de las diatomeus se ha dividido para su estudio en dos sub-familias: Placochromaticeas y Coccochromaticeas, división basada en la constitución de la endocroma, correspondiendo a la primera una estructura formada por placas y a la segunda una estructura granular. Se da el nombre de endocroma a las celdillas que en las algas filamentosas continen la materia colorante; está constituída por clorofila y leucoxanteína, dominando la segunda sobre la primera y modificando el color de la cloroiila. Actualmente se conocen más de 10,000 especies. Su gran adaptabilidad a todos los medios, hace que prácticamente se le encuentre en todos los climas, agrupada en cantidades incomensurables.

Las diatomeas son algas unicelulares pertenecientes al grupo de las feoficias, orden de las bacilarias. Su constitución interna corresponde a la de una célula vegetal: perotoplasma, endocroma, núcleo, nucleolo y grupos cromatóforos. Están provistas de esque- 
leto silícico, que las distingue de los demás seres del reino vegetal. Constituídas por un caparacho a frústula compuesta de dos valvas, I $a$ superior o ventral e inferior o dorsal. Comparativamente semeja una caja con sus tapas superior e inferior interpenetradas, forma de estructura relacionada con los procesos de reproducción. La unión o sutura entre las caras de las valvas recibe el nombre de lado conectivo.

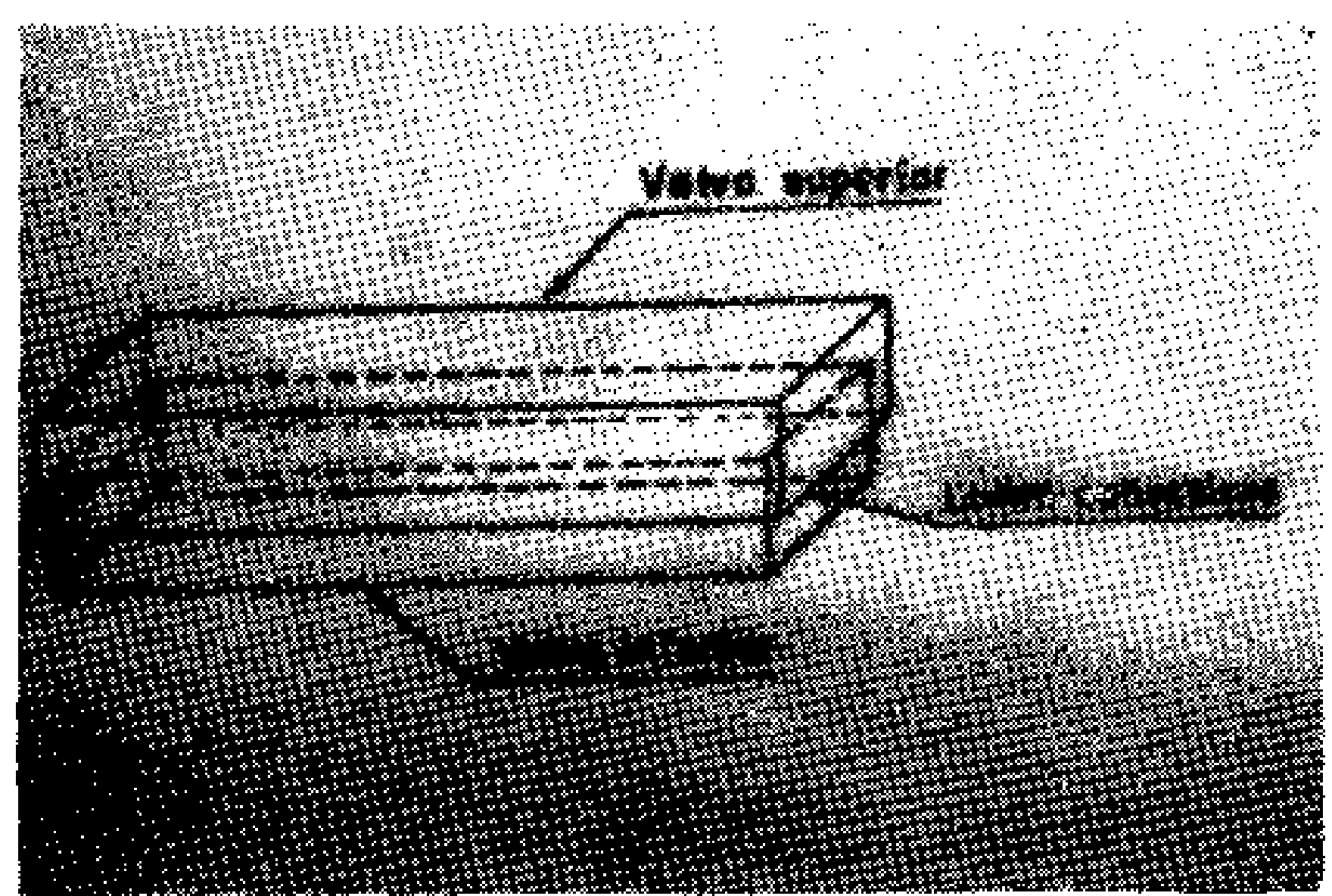

Figura No. 1.

Jas superficies valvares presentan características purticulares, las que definen cada especie o tribu, así como el género a que pertenecen. Se distinguen por su aspecto, pudiendo ser cóncavas 0 convexas y de formas muy diversas: elípticas, lanceoladas, cuneiformes, discoidales, etc.

Los nódulos son tres levantamientos que pueden distinguirse distribuídos uno en la parte central y uno en cada extremo de la. superficie valvar; el central puede alargarse longitudinal o transversalmente, recibiendo el nombre de estauro, forma que semeja una cruz con un brazo mayor que el otro. Las diatomeas pueden tener uno, dos, tres nódulos o ninguno.

Los nódulos pueden estar unidos entre sí por un bords grueso a modo de nervadura llamado rafa, con aspecto de labios, Ia que pone en contacto la parte interior con el exterior. En algunos ejemplares no se observa el borde grueso característico de la rafa, sino que sólo se observa un espacio liso $y$ angosto, donde se detienen los detalles de ornamentación; en otros, los detalles de ornamentación cubren totalmente la superficie valvar, no observándose detalle alguno que indique la existencia de la rafa. 
De los bordes de la superficie valvar hacia el centro, el ornato se distribuye en la superficie de ésta, deteniéndose hasta un poco antes de la rafa, dejando una zona lisa y limpia hacia los bordes de ésta, conocida con el nombre de zona hialina. Al borde o ceja que se observa en las uniones valvares se le designa con el nombre de carena.

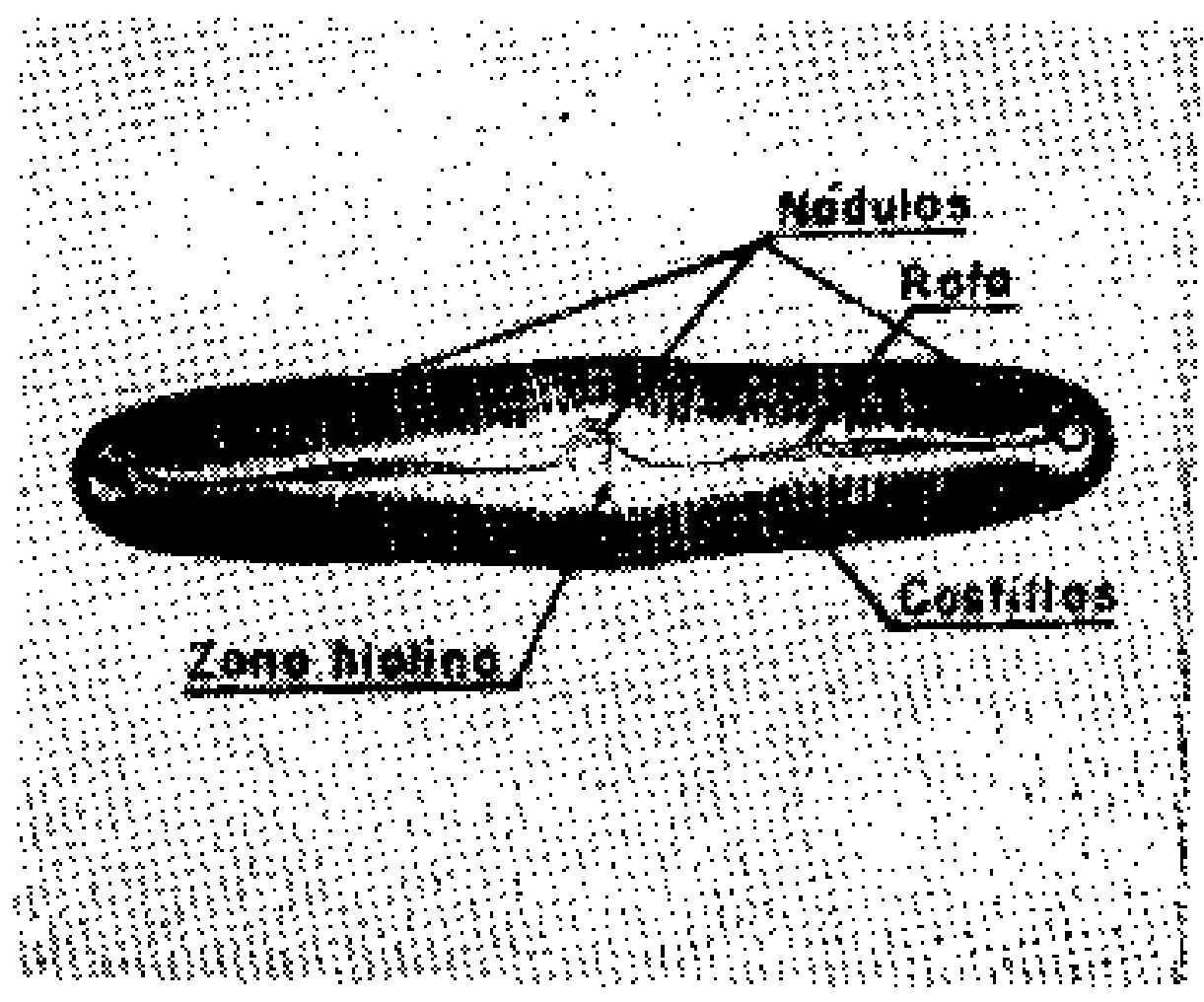

Figura No 2

La existencia o no existencia de nódulos y de rafa, la distił* bución del ornato, la forma de las superficies valvares y la unión de las frústulas, ya sea por las caras valvares o por los lados conectivos, son características que determinan y clasifican cada tipo o ejemplar de diatomea. Por ejemplo, la presencia de rafa en las

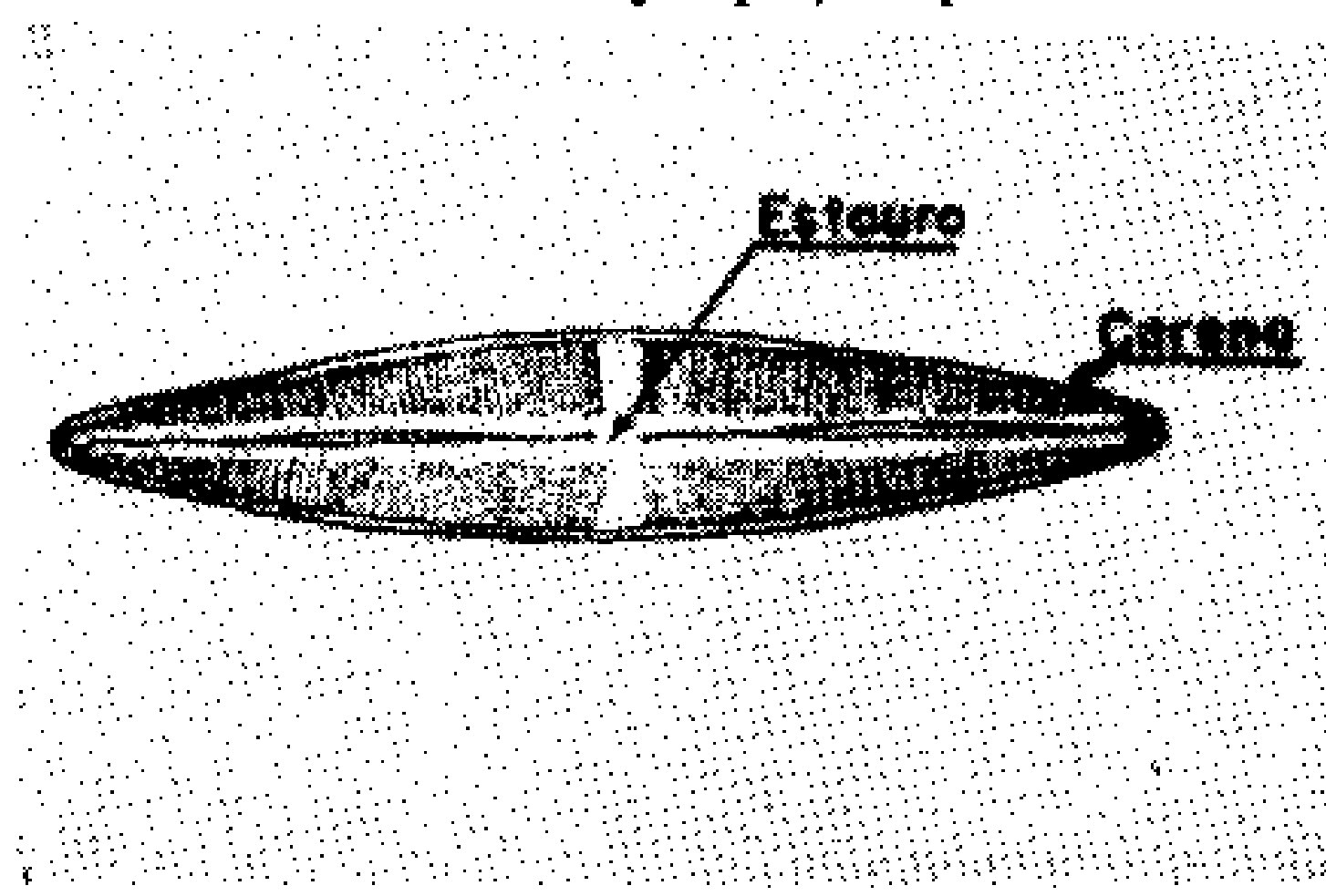

Figura No 3

diatomeas las clasifica dentro de un grupo especial, designado con el nombre de Rafidias; cuando en el lugar de la rafa sólo se observa un espacio liso y angosto, constituyen al grupo conocido como el de Pseudorafidias; la ausencia total de rafa caracteriza a] grupo de las Criptorafidias. 


\section{DEPOSITOS DE DIATOMITA EN LA REPUBLICA MEXICANA}

Son contados los estudios que se han efectuado sobre las diatomitas mexicanas, $(11,12,13,14,15)$, por lo que el autor ha tenido el deseo de contribuír al mejor conocimiento de las mismas. Las localidades registradas que indican la existencia de diatomita en el país, se encuentian registradas en los Boletines núneros 40 y 41 publicados por el Instituto de Geología; no se ronoce por el momento ningún otro dato que amplíe las localidades que a continuación se citan:

Lago de Texcoeo, Distrito Federal.

En terrenos de la Hacienda de San Andrés, Municipio de Magdalena, Estado de Jalisco.

En la Hacienda de Extipac, cerca de Santa Ana, Municipio de Coeula, Estado de Jaliseo.

Valle de Toxi, Municipio de. Ixtlahuaca, Fstado de México.

Tajo de Nochistongo, Municipio de Huehuetoca, Estado de México.

Boca del Viento, Municipio de Sultepec, Estado de México.

Excavación para la desviación del río de Tlalnepantla y los Remedios, entre los pueblos de San Juan Ixhuatepepec y el Rancho de Santa Cruz, Municipio de Tlalnepantla, Estado de México.

Faldas del cerro de Soltepec, cerca del pueblo de Purificación, al oriente y a corta distancia de la Ciudad de Texcoco, Estado de México.

Municipio de Nicolás Romero, Estado de México.

Zacapu, Municipio de Zacapa, Estado de Michoacán.

La mayor parte de la diatomita que se ha consumido en México provino do Ixtlahuaca, Tlkinepantla y Texcoco, Estado de Máxico. En el año de 1918, se descubrieron los yacimientos que se localizan en el Municipio de Tlaxcala, Estado de Tlaxcala, únicos yacimientos que a la fecha están siendo explotados técnicamente y con grandes éxitos.

\section{MUESTRAS ESTUDIADAS}

El que esto escribe tuvo la oportunidad de estudiar dos muestras de diatomitas procedentes de los Municipios de Nicolás Romero y de Tlalnepantla, y dos muestras más, procedentes del Estado de México.

MUESTRA No. 1.-Yacimiento de Villa Nicolás Romero.La Villa Nicolás Romero, perteneciente al Municipio de Nicolás Romero, Estado de México, está situada aproximadamente a 30 kilómetros de la Ciudad de México, saliendo de Azcapotzalco por la carretera que va a Tlalnepantla, y de aquí siguiendo la desvia- 
ción que pasa por Atizapán y Nicolás Romero, para terminar en Progreso Industrial, villa con población aproximada de 5.000 habitantes, los que dedican sus actividades prineipaImente a la agricultura. parte de ellos siendo empleados en las fábricas de Barrón. La Colmena y otras.

Los yacimientos se encuentran en terrenos cercanos a la fábrica La Colmena, constituyendo una propiedad privada donde sus moradores han edificado sus habitaciones. Jos depósitos son de dimensiones variables, cubiertos por capas de cenizas volcánicas, toba voleánica $y$ tierra vegetal. Desde hace algunos años se han venido explotando por los moradores de la región, abriendo pequeños socavones de 10 metros de profundidad, con lo que ponen al descubierto las capas de diatomita que extraen y almacenan a la orilla de la carretera en espera del comprador ocasional. La diatomita se presenta en forma de mantos con espesores que varían entre $60 \mathrm{~cm}$ y $80 \mathrm{~cm}$. El suscrito aún no ha tenido la opnrtunidad de hacer un franco reconocimiento del área donde sa localiza la diatomita, por lo que desconoce sus posibilidades.

MUESTRA No. 2.-Municipio de Tlalnepantla. Estado de Mèxico.-Muestra proporcionada por el señor Juan Díaz vecino de la localidad. Vértiz.

Muestras números 3 y 4.-Proporcionadas por el señor Félix

DESCRIPCION Y OBSERVACIONES MICROSCOPICAS.-

Todas las muestras tienen una particularidad en común que las identifica de inmediato: su poco peso, en relación con el volumen. Las variedades más puras se distinguen por su color claro; a la menor presión se disgregan en polvo fino que, al manipularse entre los dedos, recuerdan la sansación que produce el talco. A medida que aumenta el número de impurezas, pues los mantos se encuentran intercalados entre tobas y cenizas volcánicas, el material adquiere un color que varía desde el gris cerizo hasta el gris característico de los limos areillosos, presentando los terrones y grumos mayor dificultad para ser disgregados, lo que aún puede hacerse sin gran esfuerzo, prevaleciendo la sensación del talco, quedando como residuo pequeños gránulos y grumos de tierra vegetal. arcikla o toba. El color de las muestras al ser molidas se aclara ligeramente, teniendo las números 3 y 4 color blanco de veso, la número 2 gris claro y la número 1 , gris típico. A pesar de que la muestra número 1 es la más obscura de todas, al ser calcinada a la temperatura de $400^{\circ} \mathrm{C}$. por treinta minutos adquirió el color claro de las muestras númeron 2,3 y 4 .

La observación microscópica permite identificar el mismo tipo de individuos en cada una de las muestras; un detalle característico que se observa a primera vista en la muestra No. 1, es 
la abundancia de Naviculeas de tamaño gigante, si se comparan con los tamaños dominantes en las demás.

Las distintas especies o tribus que han sido identificadas en las diatomitas mexicanas, estudiadas por el masstro Enricue Díaz Lozano, son las siguientes, (16): Como pertenecientes a la subfamilia de las Placochromaticeas; Achnantheas, gomphonemeas, cymbeleas, Naviculeas, Nitzachieas, Surireleas, Sinedreas, y Eunotíeas. Como pertenecientes a la subfamilia de las Cocochromatíceas se citan las Fragilareas y las Gailoneleas.

Achnantheas.-Se caracterizan por estar constituidas por dos valvas, una cóncava y la otra convexa; la primera se encuentra provista de un nódulo en forma de estauro y rafa, lo que la diferencia de la valva convexa superior, la que posee una pseudorafa y carece de nódulos. Es de forma elíptica, naviculoide y discoidal. En las preparaciones se observaron las formas elíptica y raviculoide.

Cymbeleas.-Caracterizadas por tener caras valvares simbiformes y simétricas con respecto a su eje menor. Están provis. tas de rafá eon un nódulo central y dos terminales.

Naviculaas.-Caracterizada por sus frústulas, las que tiener: sus caras valvares simétricas a los dos ejes. Poseen un nódulo central y ocasionalmente dos terminales.

Surireleas.-.-Se caracterizan porque las frústulas tienen sus caras simétricas. De forma elíptica, ovoide, redondeadas. Poseen una pseudorafa y carecen de nódulos. Las frústulas poseen una ẹrena más o menos marginal, observándose como pequeña saliento en las uniones valvares.

Sinedreas.-Se caracterizan porque Ias frústulas son alargadas, en forma como de bastones o reglas y de sección eilíndrica, con un nódulo en forma de pseudoestauro y pseudorafa.

Fragilaraas.-Se caracterizan por tener forma elíptica o lancolada, carecen de rafa, nódulos y carena. Las valvas se unen por sil cara valvar para formar cintas.

Gailoneleas.-Se caracterizan porque las frústulas al agruparse tienen formas cilíndricas o elipsoidales, se encuentran unidas por sus caras valvares formando largas cintas. Las caras elipsoidales son planas, globulares o elíptcas. Observadas por su cara valvar afectan formas cilíndricas alargadas, semejando fracciones do tubo. Les microfotografías muestran el tipo general de diatomeás que constituyen las muestras estudiadas. Los factores forma y tamaño sugieren brillantes posibilidades para el empleo como material filtrante o como ayuda para filtración. La complicáda estructura que caracteriza a estos microorganismos forma miles de pequeñas celdillas o cámaras de aire, a las que debe su gran porosidad, la que proporciona un excelente medio de filtración. Su 
gran superficie y baja densidad aparente, son características que rigen el poder absorbente, decolorante y clarificante.

ANALISIS QUIMICO Y DETERMINACIONES.-A continuación, en forma tabulada, se presenta el análisis químico $\mathrm{y}$ otras determinaciones efectuadas en las muestras.

Muestra No.

Determinaciones

$$
\mathrm{SiO}_{2}
$$

$\mathrm{Fe}_{2} \mathrm{O}_{3}$

$\mathrm{Al}_{2} \mathrm{O}_{3}$

$\mathrm{CaO}$

Humedad $\mathrm{y}$

perd. $\mathrm{x}$ calcinación

Indeterminados

Total

$\mathrm{pH}$
1

\begin{tabular}{r}
78.26 \\
0.76 \\
3.30 \\
1.80 \\
\\
8.29 \\
7.64 \\
\hline 100.00 \\
7.9
\end{tabular}

2

84.32

0.80

1.50

0.35

9.76

3.39

100.00

8.4
3

85.43

0.92

2.58

2.54

7.13

1.40

100.00

7.2
4

81.93

1.25

5.48

0.32

7.26

3.76

100.00

7.3

Poder de absorción

$\begin{array}{lllll}\text { Inerte } & 63 \% & 50 \% & 45 \% & 45 \% \\ \text { Kerosina } & 37 \% & 50 \% & 55 \% & 55 \%\end{array}$

Muestra No.

1

2

3

4

Determinaciones

Tamaño de las diatomeas

en micras

Máximo

333

Mínimo

Dominante

33

142

299

23.5

71

93

14

152

Grados de molienda

recomendables

$\begin{array}{llrrrr} & \text { Mallas } & 100 & 200 & 270 & 270 \\ \text { Máxima } & \text { Micrones } & 149 & 74 & 53 & 53 \\ & \text { Mallas } & 40 & 50 & 150 & 100 \\ & \text { Micrones } & 420 & 295 & 105 & 149\end{array}$

El análisis químico de las muestras presenta como características principales contenido alto de sílice, contenido bajo de fierro total, humedad y pérdidas por calcinación. El pII de cada una de las muestras se encuentra dentro de los límites señalados pon los industriales para el aprovechamiento de la diatomita como portador de insecticidas. Su poder de adsorción se clasifica como bueno, existiendo la posibilidad de que éste pueda aumentarse por calcinación adecuada. 
Es de absoluta importancia estudiar cuidadosamente el grado de molienda a que debe sujetarse el material, con objeto de tener el mayor porcentaje de diatomeas completas, ya que esta es una de las características que tienen notable influencia sobre el poder de filtración. Si se desea efectuar la molienda sin destruir diatomea alguna, el grado de molienda a que se debe sujetar cada una de las muestras deberá ser de $40,50,150$ y 100 mallas respectiva. mente; un sistema adecuado de clasificadores de ciclón proporcionará los distintos tamaños que se requieran. Siendo los tamaños dominantes de las diatomeas para cada una de las muestras el de $142,71,40.5$ y 52 micras, no se recomienda efectuar una molienda superior a $100,200,270$ y 270 mallas, pues con esto se rompería la estructura de la mayor parte de las diatomeas. Los productos industriales para filtración generalmente han estado sujetos a una molienda mínima de 150 a 200 mallas, aprovechándose los distintos tamaños obtenidos al efectuar su clasificación.

El trabajo de control ha sido incompleto debido a la falta de equipo de molienda y de clasificadores adecuados, así como de otros elementos, por lo que no ha sido posible determinar el poder filtrante, grado de porosidad y su máximo poder de adsorción.

En el trabajo presentado por el señor Víctor Félix, sobre la Diatomita en México, 17 presenta los resultados obtenidos en distintas pruebas de comparación efectuadas con tierras diatomáceas procedentes del Éstado de Tlaxcala y tierras diatomáceas industrializadas de importación. Los resultados muestran que Ia diatomita del Estado de Tlaxcala es de calidad superior a diatomitas que venden los representantes de la Johns Manville en México, superior como material aislante y superior como material filtrante. A últimas fechas se tuvo la oportunidad de comparar en forma imperfecta algunas muestras del material que vende la casa Johns Manville de México, obteniéndose resultados muy halagadores.

EXPLOTACION. - La explotación dirigida técnicamente en este tipo de yacimientos es nula; se concreta a la formación de socavones de mayor o menor tamaño, trabajándose con pala para desprender bloques o pedruzcos, amontonándolos a la puerta de los socavones y llevados en costales hasta el lugar de almacenamiento. Como se indicó al principio, la explotación de estos materiales es sumamente rudimentaria.

Tomando como base los trabajos que con este objeto se desarrollan en algunos depósitos de. la Unión Americana, 18, 19, 20 se menciona lo siguiente: La explotación generalmente se desarrolla a cielo abierto, empleando diversos elementos mecánicos para eliminar la tierra vegetal y las diversas capas que cubren el yacimiento, dejando libre el depósito; el material es llevado a una quebradora o molino primario, donde sufre desintegración inicial, pasando después por una criba vibratoria, obteniéndose así el ma- 
terial desintegrado de diversos tamaños, según el número de cribas que se hayan instalado. Para determinadas aplicaciones industriales es suficiente la desintegración antes indicada, por lo que puede empacarse e inmediatamente salir al mercado. Es de recomendarse, como instalación mínima, un equipo constituído por una quebradora 0 molino desintegrador inicial, secador rotativo para eliminar la humedad que tiene variaciones entre 9 y $25 \%$; un segundo molino, el que se recomienda que sea de discos o de rodi: llos, recubiertos con hule duro para evitar el rompimiento de las frústulas. Según los informes obtenidos, hay instalaciones que tienen en sus circuitos dos molinos de martillos, usados como moledores primario y secundario, no sabiendo si hay o no rompimiento de las diatomeas con este tratamiento tan drástico.

Instalaciones más avanzadas tienen en su circuito cuatro clasificadores de aire, con lo que se obtiene selección do tamaños perfectamente dirigida. Las partículas que constituyen los diferentes tamaños del polvo así obtenido, son identificadas y registradas cuidadosamente, ya que de este registro dependen las propiedades y empleo.

Otra etapa en la industrialización de la tierra diatomácea, es la calcinación a temperaturas diferentes, calcinación que modifica las propiedades físicas del material.

Una última etapa comprende tratamientos químicos que tienen por objeto incrementar las propiedades naturales de las tierras diatomáceas, modificarlas parcialmente o proporcionarles una nueva característica para un determinado proceso industrial. Los productos así obtenidos se encuentran ya preparados y listos para su empleo.

REGULACION TECNICA.-El equipo de regulación que poseen las grandes compañías norteamericanas y europeas requiere personal técnico especializado, el que ejeree una inspección rigurosa, que se inicia en el yacimiento y termina con la inspección del material de embarque; la organización de los elementos dentro del circuito de trabajo, así como el control técnico del material, son en su mayoría datos confidenciales que reserva para sí cadal una de las compañías.

La regulación en el yacimiento consiste en la toma de muestras para determinar las mejores zonas del yacimiento, obteniendo así material de trabajo con especificaciones regulares. Las muestras son estudiadas y clasificadas rigurosamente, conociendo las características de cada zona del yacimiento, Io que asegura la producción industrial precisa y uniforme, así como un rendimiento de gastos favorable a la compañía.

Para asegurar una producción perfectamente apegada a las rigidas normas establecidas que definen el empleo industrial de los productos obtenidos, es necesario un laboratorio moderno que 
incluye los siguientes elementos: Un pequeño aeroclasificador, quebradoras y moledoras de laboratorio, mallas o cribas tipo y microscopio, además del equipo personal de laboratorio para las determinaciones de densidad, volumen, poder decolorante, poder adsorbente, poder absorbente, poder filtrante, pérdidas por calcinación, $\mathrm{pH}$, fragilidad de las partículas $\mathrm{y}$ poder abrasivo.

\section{USOS INDUSTRIALES}

El empleo industrial de las diatomitas está íntimamente relacionado con el tamaño y estructura de las diatomeas, las que ejercen su influencia directamente sobre la porosidad y textura del material. Las especificaciones son numerosas $y$ varian de acuerdo con el empleo a que se les destine; generalmente el consumidor solicita de Ia compañía que proportiona la diatomita un tipo o grado especial de material que se adapte a las necesidades de su industria; la compañía se encarga de sugerir el tipo y grado más adecuado, proporcionando las especificaciones correspondientes.

Una clasificación general del consumo de diatomita, de acuerdo con su empleo, es la siguiente: Como material filtrante, 57\%; Material inerte, 28\%; Material aislante, $8 \%$; Otrus aplicaciones, 7 por ciento.

A continuación se presenta un resumen de los principales usos de la tierra diatomácea o diatomita, con las características que deben regularse:

1.-Agente de filtración, clarificación y decoloración.

Con este objeto se requiere una estructura microscópica determninada, tamaño, mayor grado de pureza y completa inactividad ante las reacciones químicas, grado de finura, baja densidad y sobre todo un alto grado de filtración, el que se aprecia por la claridad del filtrado.

Se emplea como filtro ayuda y como medio filtrante en la clarificación de todo tipo de líquidos, orgánicos e inorgánicos, preparado en forma de losetas, placas de diferentes formas y con distintos grados de finura, crudo o beneficiado. En la filtración de soluciones siruposas de las industrias azucarera, cervecera, de vinos y licores destilables, jugos de frutas y bebidas de todas clases, aceites vegetales, minerales, animales, grasas, ceras, lacas, aceites lubricantes, alquitranes, extractos, curtientes, gases, ácidos, soluciones colorantes $\mathrm{y}$ decolorantes, aguas potables $\mathrm{y}$ aguas negras, reactivos $y$ soluciones metalúrgicas, para filtraciones bacteriológicas, penicilina, estreptomicina, vitaminás, etc.

2.-Como material inerte.

Para ser empleado como material inerte requiere alto grado de pureza química y pureza de material, alto grado de inactividad, regulación del tamaño de partícula, estructura microscópica, 
grado de finura, poder de adsorción, grado de acidez ( $\mathrm{pH}$ ) y contenido mínimo de ión ferroso.

Se emplea en forma pulverizada como agente absorbente $y$ adsorbente de liquidos desinfectantes (absorbe de 150 a 200 veces su peso en agua, sin cambiar de forma), portador ideal que produce efectos lentos de mayor efectividad, portador de catalizadores en la industria química, portador de emanaciones radioactivas, colorantes, ete., etc.

3.-Como material aislante del calor y del sonido.

Requiere peso específico mínimo aparente determinado en iosetas $y$ cn polvo impalpable, conductividad térmica y regulación del grado de humedad, estructura microscópica y tamaño de grano.

Se emplea como aislante térmico en forma de ladrillos y losetas en las plantas de metalurgia, manufactura del vidrio, en hornos y varios equipos de cerámica, equipos generadores de gas, tuberías de vapor sobrecalentado, calderas, equipos para tratamiento térmico, alambiques para refinación de aceites, instalaciones generales para frío o calor, construcción de frigorificos. Como aislante del fuego, frío, calor y humedad en la construcción de pisos y paredes en los edificios modernos, ete.

4.-Otras aplicaciones.

Como agente llenador, empleado en forma pulverizada en pinturas y barnices, plásticos, hule, papel, discos fonográficos, linóleos, jabones, cerillos, tejidos para empaques incombustibles, techos, productos farmacéuticos, etc., ete.

Como agente abrasivo en forma pulverizada en jabones y polvos limpiadores, pastas limpiadoras y pulidores para metales y vi. drios, pastas y polvos dentales, ete.

Como agregado para concreto en materiales para construcción, empleándose en forma de bloques o pulverizado en cementos especiales resistentes al agua salada, enyesados, papel tapiz, etc.

PRECIOS.-Los productos de importación hechos con diatomita tienen un precio variable en el mercado nacional, que oscila aproximadamente alrededor de $\$ 1,000.00$ tonelada. El mercado americano para los diversos productos tiene precios comprendidos entre DIls. 20.00 y 50.00 ; me permito mencionar que en algunas localidades del país, donde se explota diatomita en forma rudimentaria, no se pagán más de $\$ 80.00$ por tonelada.

El mercado nacional probablemente ascienda a más de 1,500 toneladas anuales como consumo exclusivo de los ingenios; a este consumo se debe agregar el de las cervecerías y las industrias de insecticidus, construcciones, pinturas y otros mereados nacionales y extranjeros que muy bien podrían conquistarse. Tomando como base un consumo anual medio de 1,250 toneladas, con precio mínimo de $\$ 800.00$ por tonelada, se obtendría un movimiento mínimo anual de $\$ 1,000,000.00$. 


\section{CONCLUSIONES.}

Por la situación tan especial que el país atraviesa $\mathrm{y}$ tomando en cuenta la poca atención que se ha dedicado al conocimiento y explotación de los minerales no metálicos, sería ideal que se planearan trabajos a manera de tratados, en los que se desarrolla. ran los capítulos correspondientes a la geología, geología económica $y$ el aspecto industrial de un determinado mineral no metálico. La presentación de esta clase de trabajos alentaría sin duda al industrial, el que percibiría nuevas oportunidades que tal vez desconoce, o que por falta de estudios adecuados no se atreve a atacar.

Se han señalado en nuestro país un buen número de localidades de tierras diatomácess de las que se desconocen por completo sus posibilidades, por lo que sería conveniente definir su valor económico; estudiarlas desde el punto de vista geológico, paleontológico e industrial.

Las cuatro muestras estudiadas son muy semejantes entre sí. ya que en todas ellas se encuentran individuos correspondientes a las tribus denominadas Achnantheas, Cymbeleas, Naviculeas, Surireleas, Sinedreas, Fragilereas y Gailoneleas.

Las cuatro muestras presentan las características que las definen como materiales aprovechables industrialmente. Por el tipo de organismos y por la estructura que los caracteriza, tienen grandes posibilidades para ser empleados como materiales filtrantes $y$ como portadores, que podrían ser utilizados en bruto, teniendo como único tratamiento una molienda adecuada; sin embargo, es de recomendarse que no se escatime dinero alguno y se tome n cuenta para la instalación industrial un horno primario secador, un molino primario desintegrador, equipo adecuado y cribas para eliminar impurezas, un horno secundario de calcinación, dos clasificadores de ciclón para tamaños comprendidos entre 150 y 250 mallas y para partículas comprendidas entre 250 y 325 mallas.

Las muestras números 1 y 2 quedan clasificadas tentativamente como tierras diatomáceas de mediana calidad; las muestras números 3 y 4 como tierras diatomáceas de buena calidad.

Para definjr el valor exacto de los yacimientos y las aplicaciones industriales que pudieran tener, sería necesario un estudio geológico económico y un mejor dominio de laboratorio.

Es de recomendarse a los industriales que dirijan su atención a la explotación e industrialización de las tierras diatomáceas, mineral no metálico de grandes posibilidades para el futuro.

Se podrían iniciar los trabajos con una instalación y laboratorio para trabajos en pequeña escala, instalación que se aumentaría conforme los recursos económicos lo permitieran y la necesidad lo exigiera. 
$B I B L I O G R A F I A$

1 -Las Diatomitas Mexicanas, por Enrique Díaz Lozano. Anales del Instituto de Geología de México, Tomo I, No. 1. 1917.

2 - Es ampliamente conocido el empleo de la diatomita como material de construcción, el que al ser mezclado con el cemento produce un material de considerable resistencia.

3- Los denominaban Tizatl, Tizate o Tízar, nombres empleados aún por los indigenas para denominar el material de que se trata. Se dice que un emperador Tlaxcalteca construyó un templo de Tizatl.

4 - Diatomite, por A. B. Cummins y Henry Mulryan, Industrial Minerals and Rocks, publicado por The American Institute of Mining and Metallurgical Engineers, New York, 1949.

5 - Diatomite, por Arthur F. Taggart, Hand Book of Mineral Dressing, 1950.

6-Diatomite or Diatomaceous Earth, Non Metallic Mine por Raymond B. Ladon y W. M. Myers. Me Graw Hill Book Co., Inc., 1951.

7 - Para algunos usos, las propiedades químicas no son muy importantes, exceptuando las impurezas que aumentan la densidad aparente $y$ dan coloración al producto natural o calcinado. Para otros usos se requiere material exento de impurezas, las que afectan la fusibilidad o la solubilidad en los diferentes reactivos; en ocasiones las impurezas introducen substancias indeseables en cualquier otro producto.

8-Les Diatomees, por el Dr. J. Pelletan, 1881, París, Francia.

9 - Bibliografía citada en el No. 1.

10 - Depósitos Diatomíferos en el Valle de Toxi, Ixtlahuaca, Estado de México, por Enrique Díaz Lozano. Instituto de Geología, Anales en 4o., Tomo I, No. 9.

11 - Sinopsis Mineralógica, por el Ing. Carlos F. Landero, 1888.

12 - Bibliografía citada en los números 1 y 9.

13 - La Diatomita en México, por el señor Víctor Félix. Trabajo presentado en la Primera Convención Interamericana de Recursos Minerales, 1951. 
14 - Catálogo Sistemático de las Especies de Minerales de México. Instituto de Geología, Boletín No. 40, 1923.

15 - Catálogo Geográfico de las Especies Minerales de México, Instituto de Geología, Boletín No. 41, 1923.

16 - Bibliografía citada en los números 1 y 10.

17 - Bibliografía citada en el No. 13.

18 - Diatomaceous Deposits of Northern Santa Bárbara, County; Cal., por Arnold Rand Anderson, Department of the Interior, United States Geological Survey, Bull. No. 315, 1907 , p. 438.

19 - Diatomite from Clark, Nevada. Trade Literature of Eagle Pitcher, Co.

20 - Diatomites of the Pacific Northwest as Filler Aids, por Skinner, K. G. y otros, U. S. Bur. of Mines, Bull. No. 460, 1944.

21 - Mineral Year Book 1950, Bureau of Mines, U. S. Department of Interior, U.S.A. 


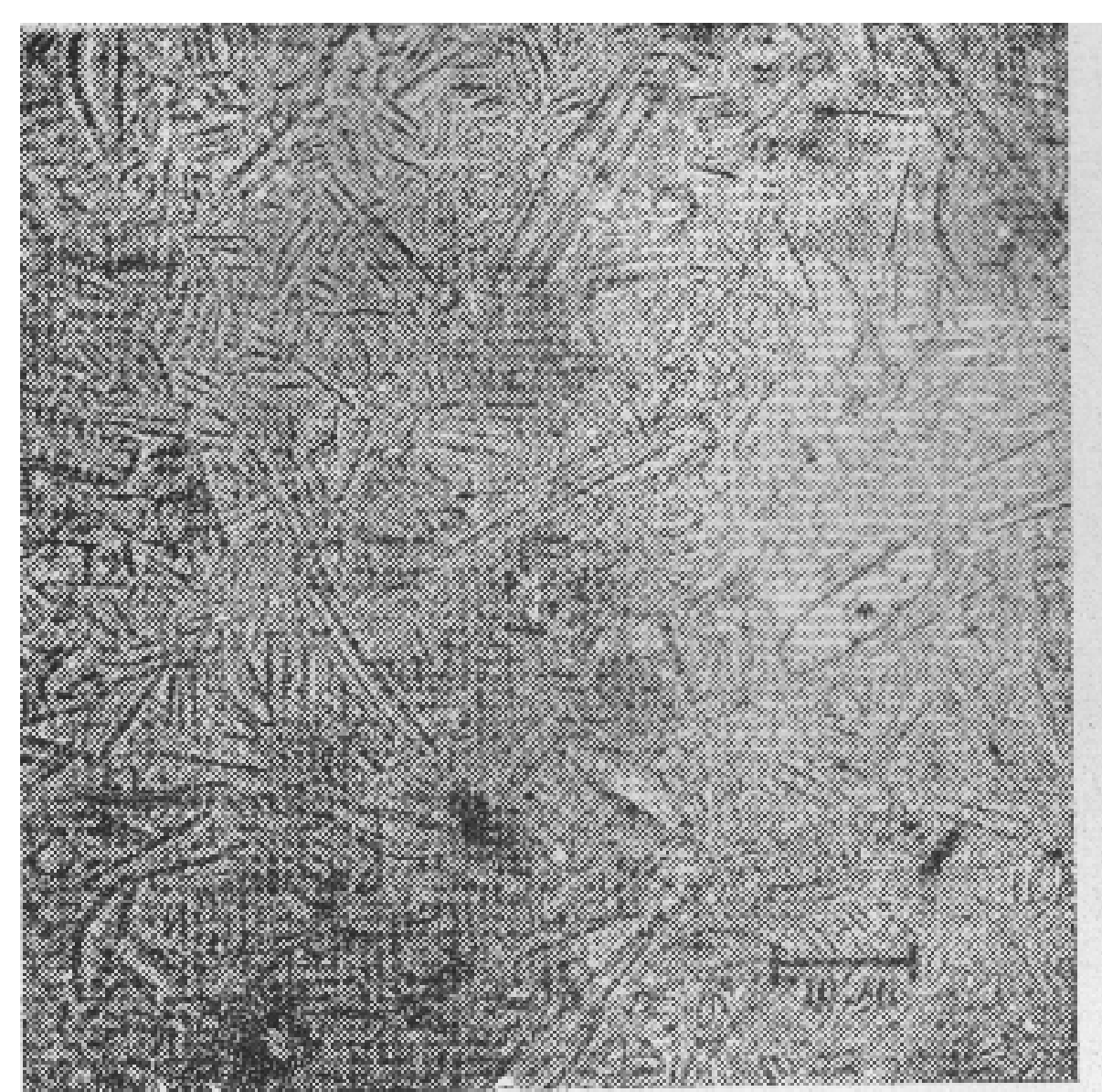

No. 1

Achnantheas, Cymbeleas, Naviculeas Sinedreas, Fragilareas.

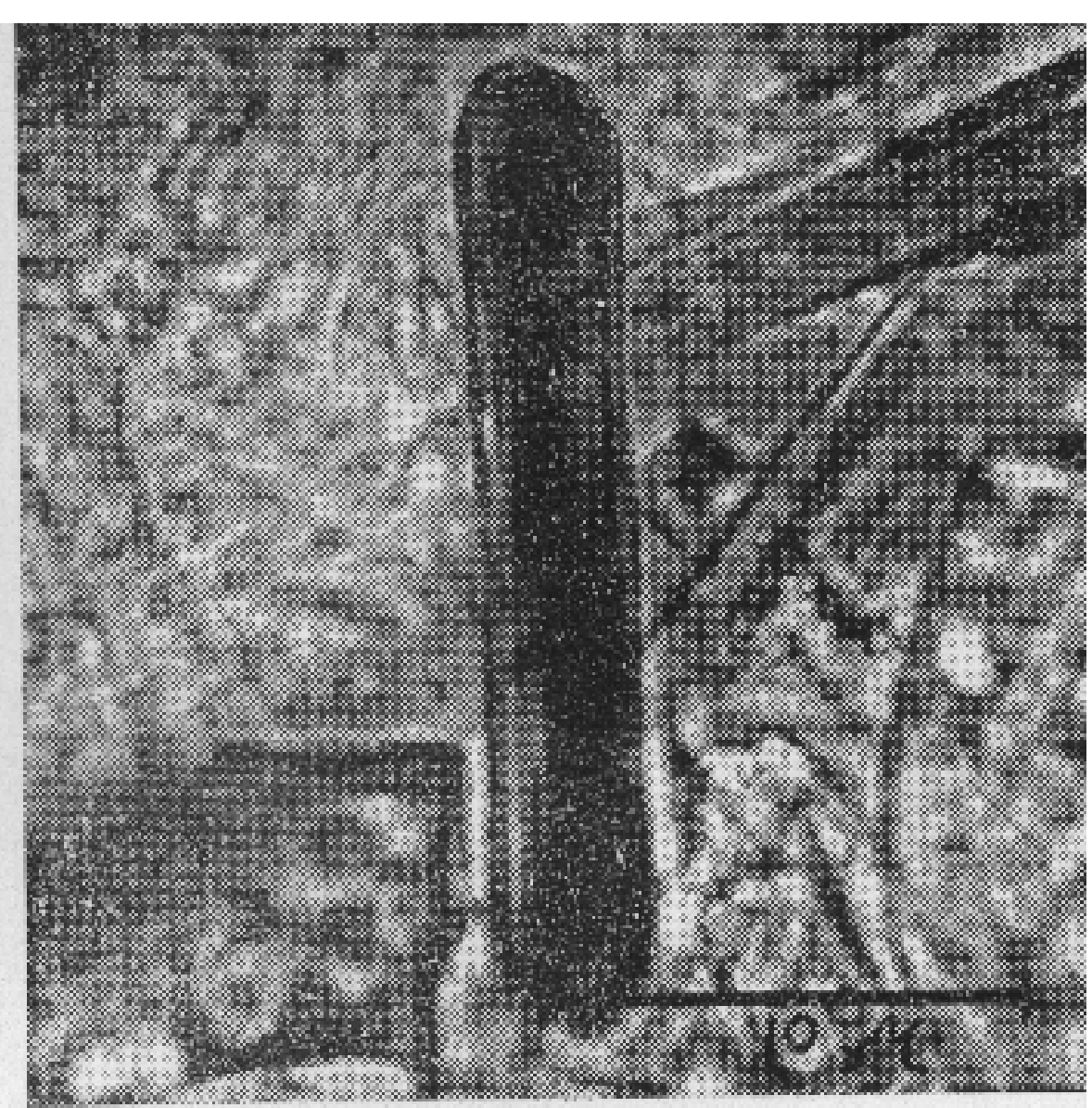

$\mathrm{N} c$ i $\mathrm{A}$

Naviculeas, Gailoneleas

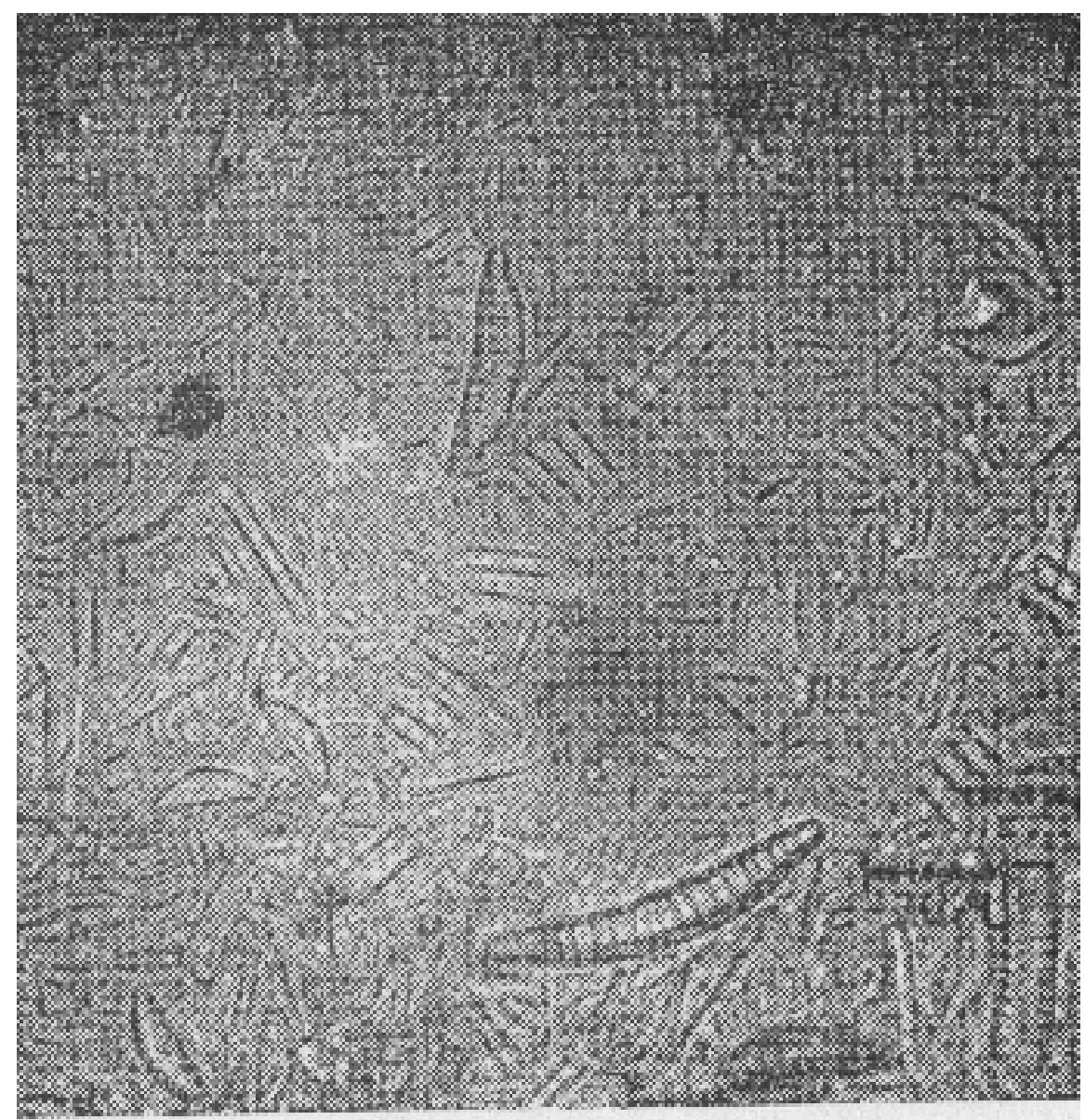

No. 2

Achnantheas, Cymbeleas, Fragilareas. 


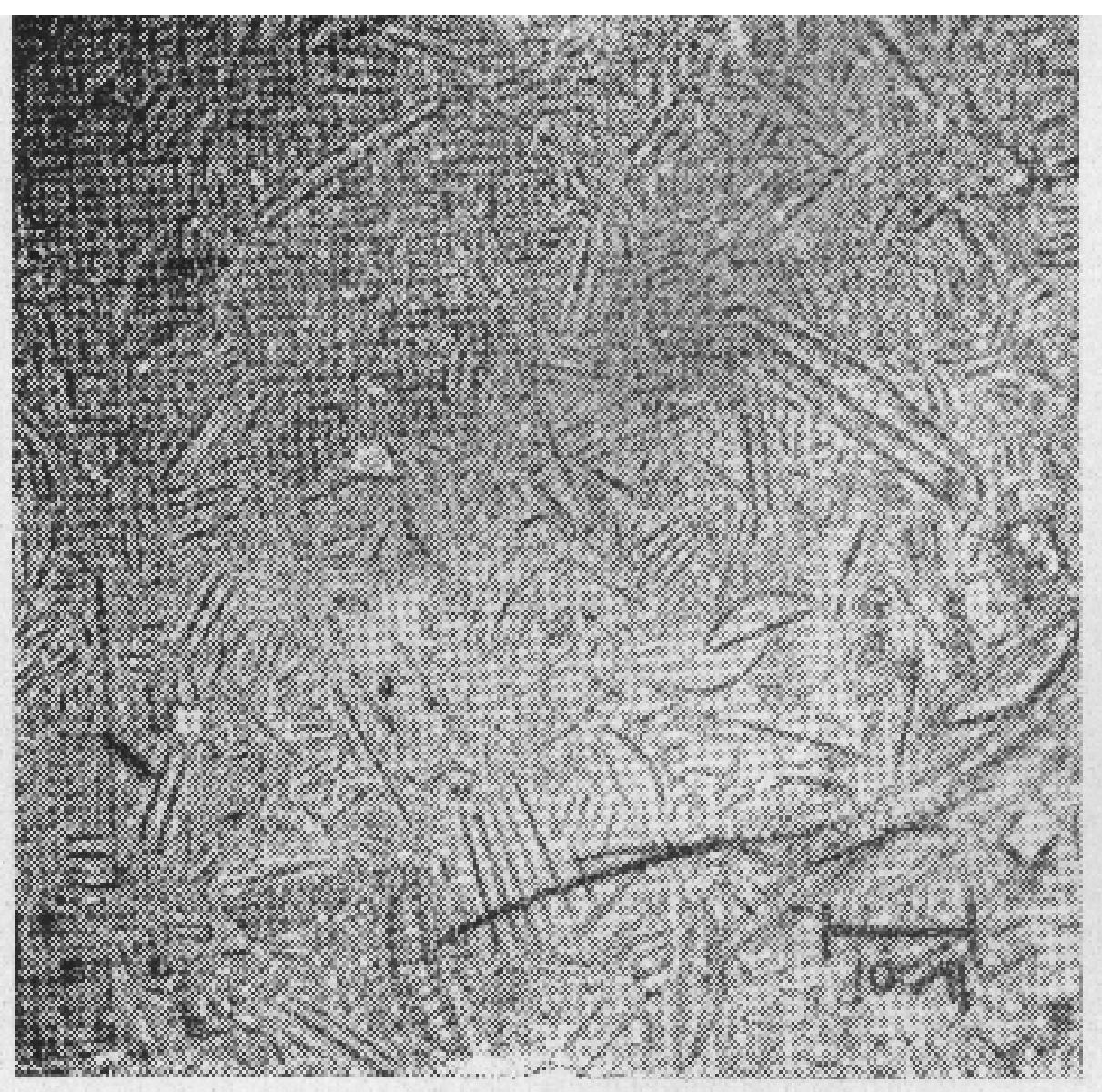

No. 3

Cymbeleas, Achnantheas, Naviculeas, Synedreas.

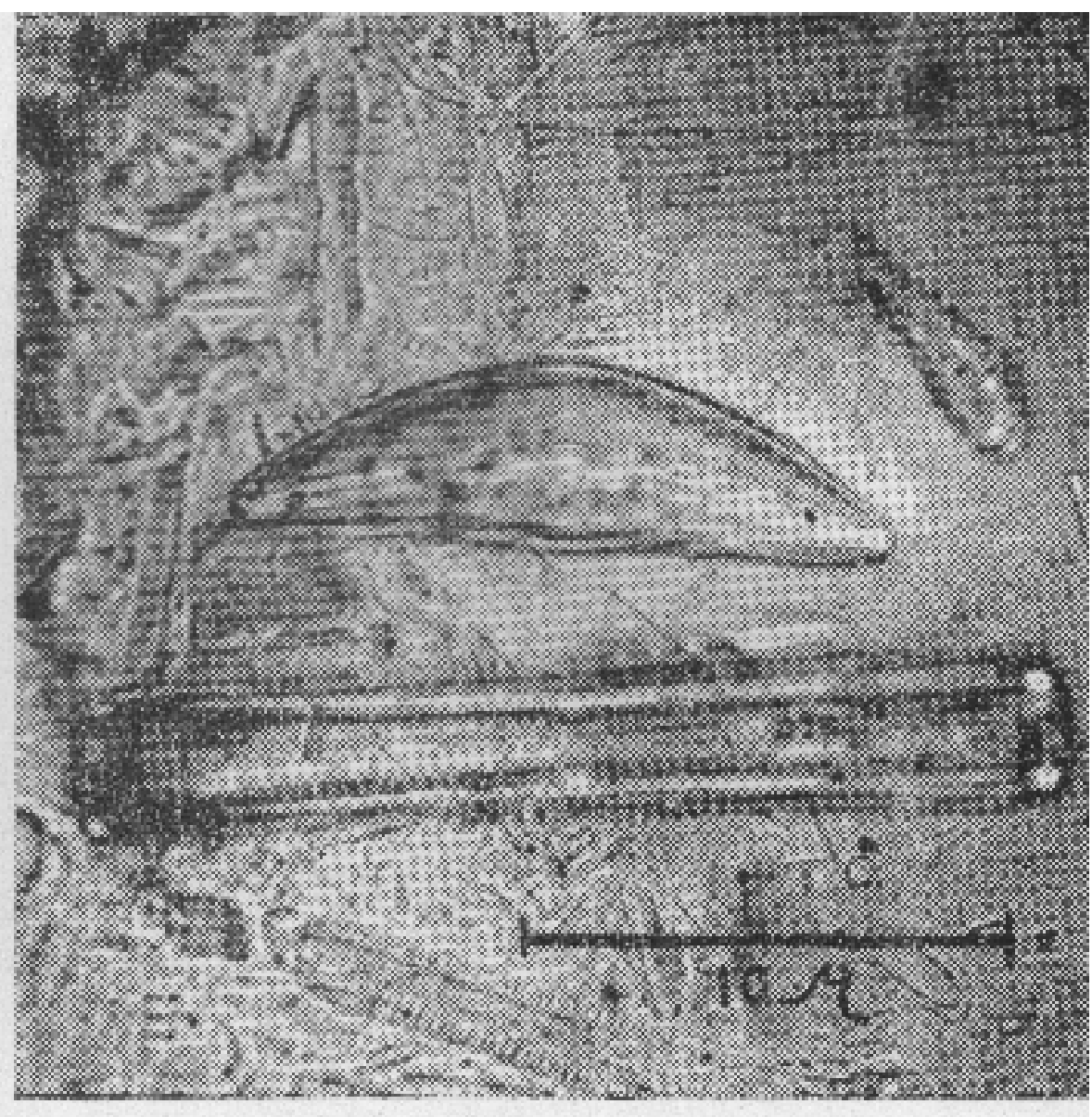

No. $3 \mathrm{~A}$

Cymbeleas, Naviculeas, Synedreas

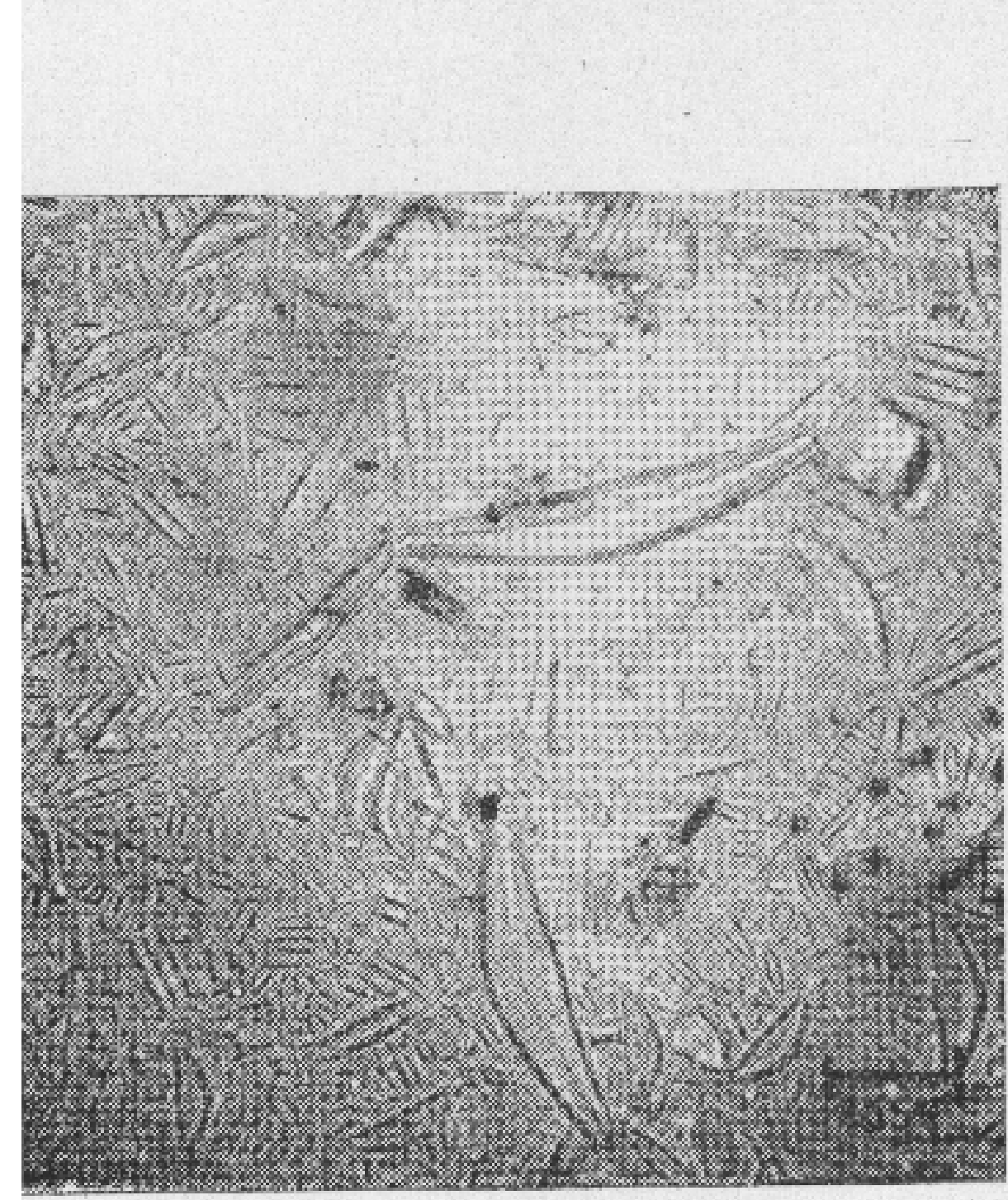

No, 4

Cymbeleas, Achnantheas, Navicule s, Synedreas. 